values $<0.05$ were considered significant. Due to between group baseline differences, a post-hoc subgroup analysis of neonates born at $\geq 35$ weeks gestation was performed.

Results The MST group had significantly lower birth weights and gestational ages at birth than the SNT group. Baseline characteristics were similar between the $\geq 35$ weeks gestation MST and SNT sub-groups.

MST had a higher successful insertion rate $(72 \%$ vs $40 \%$; 95\% CI for difference $10-50 \% ; \mathrm{p}=0.0046)$ and first pass success rate $(53 \%$ vs $26 \%$; $95 \%$ CI for difference $6-46 \%$; $\mathrm{p}=0.014$ ) than SNT. MST required significantly fewer skin breaks on average for every successful PICC than SNT (2.5 vs 5.6; 95\% CI for difference 1.15-4.90; $p=0.002$ ). The logrank test (figure 1) demonstrated a significantly higher successful insertion rates with MST than SNT as the numbers of skin breaks increased (Logrank test $\mathrm{p}=0.003$ ).

Whilst there was a higher successful insertion and first pass success rate with MST in the $\geq 35$ weeks gestion sub-group, these did not reach statistical significance. However, the logranks test reached statistical significance in favour or MST $(\mathrm{p}=0.015)$.

The cost of the disposables for one MST attempt was $£ 86.50$ for $1 \mathrm{Fr}(\mathrm{n}=10)$ and $£ 103.90$ for $2 \mathrm{Fr} \quad(\mathrm{n}=33)$ PICCs and for SNT was $£ 38.50$ for $1 \mathrm{Fr}(\mathrm{n}=4)$ and $£ 55.90$ for $2 \mathrm{Fr}$ $(n=31)$ PICCs. The total cost per successful PICC (or cost of all attempts divided by the number of successful attempts) in the study period were therefore $£ 138.51$ for MST and $£ 134.78$ for SNT.

Conclusions MST is likely superior to SNT for PICC insertion regardless of weight and gestational age at birth. The costs per successful PICC for MST and SNT in this study were similar.

\section{EPIDEMIOLOGICAL AND CLINICAL PROFILE OF CONGENITAL MALFORMATIONS IN BENGHAZI PEDIATRIC HOSPITAL}

Yasmeenah Alkharshoufi, Yasmeenah Alkharshoufi. Libya

\subsection{6/bmjpo-2021-RCPCH.36}

Background According to $\mathrm{WHO}$, the term congenital malformations can be defined as structural or functional anomalies that occur during intrauterine life and can be identified prenatally, at birth, or sometimes may only be detected later in infancy, such as hearing defects (WHO, 2016). Congenital malformations may be minor or major. The minor malformation is defined as structural abnormality present at birth, which has minimal effect on clinical function but has a cosmetic effect e.g. preauricular tag. Major malformation has significant effects on function or social acceptability e.g. CHD, CNS anomalies (NTD, hydrocephalus), digestive anomalies (abdominal wall defect and diaphragmatic hernia), (American College of Medical Genetics, 2013). The pattern and prevalence of congenital malformations vary over time and geographical location. This could be related to different detection and recording methods. The true difference in frequency may be caused by a complex interaction of known and unknown genetic and environmental factors including sociocultural, racial variables (Singh et al., 2009, Sekhobo, 2001). European Surveillance of Congenital Anomalies (EUROCAT, 2014) is a WHO collaborating center for the surveillance of congenital malformations.
Objectives The objectives were to assess the nature and the frequency of congenital malformations among infants and children who were admitted to Benghazi pediatric hospital and to study the associated maternal and neonatal risk factors.

Methods A cross-sectional study design was used for this study. It included infants and children aged (0-16 yrs.) who were admitted to Benghazi Children Hospital and who were following the genetic clinic, during the period of March 2016 to March 2017. These cases had dysmorphic features and multiple congenital malformations. A record sheet was used to collect the required data from cases.

Results The total number of patients was 81 children, $51.85 \%$ were females, $48.15 \%$ were males and infants represented $62 \%$. Cases with a history of consanguineous marriage represented $28.40 \%$. Equal proportions of mothers $(1.23 \%)$ had a history of diabetes, epilepsy and cardiomyopathy. Nearly a quarter of cases, (24.69\%) were low birth weight. Prevalence of congenital malformations (CMs) according to organs were; CVS (50.62\%), musculoskeletal (44.44\%), eye (24.7\%), limb defect (17.3\%), GIT (14.8\%), ear (14.8\%), CNS (11.11\%), urogenital (external \& internal)(11\%), cleft lip \& palate $(9.9 \%)$ and Trisomy 21 syndrome (48.1\%). Trisomy 21 syndrome have a high frequency of CHD compared to other syndromes; $67.9 \%$ had $\mathrm{CHD}, \mathrm{P}=0.01$. Most cases (90\%) died within the first year of life, $p=0.002$. Twenty-six percent of the studied population died during the study period.

Conclusions Most children with CMs and chromosomal abnormalities died within the first year of life. There is a limitation of screening tools, which could lead to imprecise genetic counseling. CM is a significant cause of morbidity and admission. The highest prevalence of CMs was CVS. Trisomy 21 syndrome was the most prevalent chromosomal abnormalities; these cases were at more risk to have CHD compared to other syndromes. There is a shortage of medical services, which applied as segmental services.

\section{DO MEDICAL APPLICATIONS HELP MEDICAL STUDENT DEVELOPMENT AND ENHANCE THEIR LEARNING DURING A PAEDIATRIC PLACEMENT?}

Edward Richardson, Edward Richardson. UK

\subsection{6/bmjpo-2021-RCPCH.37}

Background Medical apps are being increasingly used by students and doctors as an education and reference tool during their medical placements. However, it is unclear whether these apps are successful in helping student learning and development on a Paediatric placement.

Objectives To evaluate the success of medical apps currently used during Paediatric rotations and identify from the literature whether they are successful in helping student learning. Recommendations can then be made for what a future Paediatric medical app should include to be successfully implemented for medical student attachments.

Methods PubMed, Medline and Cochrane were searched using Major MeSH terms to identify papers relevant to the question. Inclusion of studies was based on whether papers included evidence of medical app usage by participants whilst on a medical placement. A survey was produced to gain insight into what medical apps were used by students and what would be expected on any future Paediatric app. These 
findings along with the literature would assist in making recommendations for this Paediatric app.

Results 27 papers were included in this review. Three papers were identified that studied apps specifically in aspects of Paediatrics. Overall, the studies showed that medical apps were an effective education tool by medical students during clinical placements. Survey results showed that medical apps were used by $43 \%$ of participants and $97 \%$ would like a Paediatric medical app to assist their learning during a Paediatric placement.

Conclusions Further long term studies are required to see the effects of medical apps in learning. More studies are also required to show how medical apps are beneficial for learning specifically during a Paediatric placement. A future Paediatric app should include concise information regarding common Paediatric conditions and have a simple design for quick use in practice.

\section{EARLY ONSET NEONATAL SEPSIS: EVALUATION OF THE KAISER PERMANENTE SEPSIS CALCULATOR FOR USE AT A TERTIARY NEONATAL UNIT IN THE UK}

Daniel Keen, Lucksini Selvadurai, Reham Hashem, Helen Gbinigie. UK

\subsection{6/bmjpo-2021-RCPCH.38}

Background The incidence of Early Onset Neonatal Sepsis (EONS) in the UK is currently estimated at $0.9 / 1000$ live births. NICE have published guidance (CG149) for treating infants based on antenatal risk factors and clinical indicators. In the UK, 13-20\% of infants are treated for suspected EONS based on CG149. This represents a significant burden, impacting on family bonding, breastfeeding, physical trauma through repeated cannulation, as well as on hospital bed status and finances. Covid-19 has compounded the issue, implementing restricted visitation disrupting the family unit. The Kaiser Permanente Sepsis Tool (KPT) was developed to determine the likelihood of infection based on multivariate analysis, has been used in the USA to successfully and safely to reduce the numbers of babies being prophylactically treated for this rare condition. Following on for their success we have now evaluated KPT for use within a UK demographic at Medway Maritime Hospital.

Objectives

1. Can KPT reduce prophylactic IV antibiotic use in well babies, when compared to NICE guideline CG149

2. Can KPT achieve this safely within our local demographic

Methods Data was collected from 62 newborn infants treated at Medway Maritime Hospital, between November 2019 and January 2020. These patients received prophylactic IV antibiotics as per NICE CG149. Inclusion criteria; $\geq 34$ weeks gestation and infants who were clinically well enough to be managed on the postnatal ward. The following data was obtained at the point of starting treatment; gestation, clinical examination, maternal peripartum temperature, rupture of membranes, maternal group B streptococcus. Subsequently peak CRP, blood and/or CSF culture results and duration of stay were recorded. Infants were classified into a risk category based on their peak CRP (Low <5, Medium 5-10, High $>10$ ). These findings were then compared to treatment recommended by the KPT (local incidence of EONS 1/1000 live births).
Results 16 infants were classed as high risk (26\%), 16 infants medium risk (27\%) and 29 infants low risk (47\%). The most common indication to treat was maternal pyrexia. Of infants screened, KPT recommended antibiotics for 2 infants (3\%); both of these were low risk. $47 \%$ of infants would have been kept under enhanced observations, with antibiotics being started if clinical symptoms developed. All blood and CSF cultures were negative. Of the infants classified as high risk, $50 \%$ of these infants would have been allocated to routine care. In the infants classified as high risk, there were no differences in the risk factors for neonatal sepsis, when compared with other risk categories.

Conclusions KPT represents a lucrative opportunity to reduce antibiotic use in well infants on the postnatal ward. However in line with similar studies, our results have highlighted that, just as with NICE CG149, is not infallible and liable to occasionally missing an asymptomatic child. Clinical vigilance is of the utmost importance and implementation of KPT would be have to marry with robust systems of neonatal observation and workforce training. A more conservative approach to EONS will invariably be associated with risk and ultimately it will be up to individual units to agree on what is acceptable.

\section{PATTERNS OF PRESENTATION OF SICKLE CELL DISEASE AT AHMED GASIM ESPECIAL IZED HOSPITAL FOR CHILDREN (2015-2016)}

Khalid AM Abdalbagi, Safaa Medani. Sudan

\subsection{6/bmjpo-2021-RCPCH.39}

Background Sickle cell disease is the commonest type of haemolytic anaemia in Sudan. It is commonly seen in those originating from western Sudan tribes, the affected children show various clinical presentations, laboratory findings, with varied complications and outcomes influenced by genetic, environmental and socioeconomic factors, which require a thorough assessment of patterns of presentations.

Objectives The aim of this study to determine the patterns of presentations of Sickle Cell Disease, in view of demographic data, clinical presentations, laboratory findings and the short outcome.

Methods This a prospective cross-sectional hospital based study, conducted in Ahmed Gasim Specialized Hospital for Children, (Khartoum North,Sudan) during the period from 1/ $11 / 2015$ to $31 / 1 / 2016$, covered all patients with Sickle cell disease, their ages were less than 18 years. Data collected by specifically designed questionnaires to fulfil the objectives of this study.

Results One hundred thirty eight patients were studied, aged between 9 months and 17 years with a mean age of 6.26 years ( \pm 4.44 SD). Nearly $93 \%$ of patients were belonging to western tribes of Sudan. The prevalence of sickle cell disease patients were $2.6 \%$ of total admission and $0.7 \%$ of the total number of patients seen in the Emergency Room in Ahmed Gasim Specialized Hospital for Children. Also anaemia due to Sickle Cell Disease comprises $43.9 \%$ of total anaemic patients. Seventy percent of patients were diagnosed during the first year of life. Painful crises were observed in 59\%, haemolytic crises seen in $20.4 \%$, sickle cell hepotpathy in $4.3 \%$, cerebrovascular accidents seen $1.4 \%$, sequestration crises in $0.7 \%$. Ninety fifth percent of patients presented with fever, $71 \%$ presented with fatigability, pain reported in $69.6 \%$, jaundice in 